\title{
Diffraction of a plane inhomogeneous electromagnetic wave by a perfectly conducting half-plane in an absorbing medium
}

\author{
Vladimir M. Serdyuk ${ }^{*}$, Joseph A. Titovitsky \\ Institute of Applied Physical Problems, Belarusian State University, Minsk, Belarus
}

\section{Email address:}

serdyukvm@bsu.by(V. M. Serdyuk)

\section{To cite this article:}

Vladimir M. Serdyuk, Joseph A. Titovitsky, Diffraction of a Plane Inhomogeneous Electromagnetic Wave by a Perfectly Conducting Half-Plane in an Absorbing Medium. American Journal of Electromagnetics and Applications. Vol. 1, No. 1, 2013, pp. 1-7. doi: 10.11648/j.ajea.20130101.11

\begin{abstract}
The Sommerfeld's problem of plane wave diffraction by a perfectly conducting half-plane is considered for the general case of an absorbing medium and an inhomogeneous incident wave, whose the constant phase planes are not parallel to the constant amplitude ones. The exact solution is represented in terms of parameters of incident wave propagation in the coordinate axes, but not in terms of angular variables, as usually. We adduce the original derivation of this solution, which use generalized functions and admits complex values for propagation parameters. Our approach is based on calculation of diffraction integrals by the method of transformations on the real axis without using a complex argument of integration. The results of diffraction field computation for the cases of an absorbing medium and of a decaying incident wave in a transparent medium are presented.
\end{abstract}

Keywords: Exact Solution, Inhomogeneous Wave, Perfectly Conducting Half-Plane

\section{Introduction}

The rigorous solution of the problem of plane electromagnetic wave diffraction by a perfectly conducting half-plane in a homogeneous transparent medium was obtained by Sommerfeld more than 100 years ago [1] (see also [2-5]). From that time, this solution has been generalized more than once to the cases of impedance boundary conditions on a half-plane [6], a perfectly transparent half-plane [7], a moving half-plane [8], on the case of half-plane embedded into bi-isotropic medium [9], and also on the case of different media disposed bilaterally along that [10]. The solution for a plane incident wave has stood duty as the bases for the solutions obtained for a vector spherical wave [11] and for electromagnetic beams [6, 12]. However, for an absorbing medium surrounding the half-plane, or for the case of a decaying incident wave, the Sommerfeld's solution is not applicable, because it is written in terms of real angular parameters, which determine direction of incident plane wave propagation and position of an observation point. In an absorbing medium, and also generally in the presence of decay, the propagation parameters of this wave become complex. The further attempts to obtain solutions for such cases are concerned with the approximate physical optics approach [13-15]. By this way, the solutions for a black half-plane [13], and for evanescent waves [14, 15], have been obtained using a complex angle of incidence. Meanwhile, such an approach cannot be admitted as constructive. Decaying field in an absorbing or transparent medium is excited usually after refraction of a wave (or after its total internal reflection) on a plane boundary with the other transparent medium, owing to that decay appears only in one spatial direction, which is orthogonal to the interface, but in parallel direction, wave amplitude does not vary. Hence, in practice, the presence of decay causes appearance of inhomogeneous waves, whose constant phase surfaces are not parallel to the constant amplitude ones [16]. Formal introducing a complex-valued angle of incidence does not allow simulation of propagation for such waves, and here, one should use the representation of diffraction solution in terms of the parameters of wave propagation in the various coordinate axes, which can be complex simultaneously or separately. In this work, such a representation is presented for the solution of the problem of electromagnetic wave diffraction by a perfectly conducting half-plane. At that, the original technique of its obtaining is used, because the standard method of obtaining of the 
Sommerfeld's solution assumes real values of propagation parameters and, strictly speaking, needs grounding to be applicable on their complex values.

\section{Solution of the Diffraction Problem}

As it is known, for two-dimensional diffraction problems in the Cartesian coordinate system, Maxwell's equations are reduced to the following relations, determining two different polarizations of field $[2,4,17]$

$H$ polarization

$$
E_{z}=u \quad H_{x}=-\frac{i}{k} \frac{\partial u}{\partial y} H_{y}=\frac{i}{k} \frac{\partial u}{\partial x}
$$

\section{E polarization}

$$
E_{x}=\frac{i}{k} \frac{\partial \bar{u}}{\partial y} \quad E_{y}=-\frac{i}{k} \frac{\partial \bar{u}}{\partial x} \quad H_{z}=\varepsilon \bar{u}
$$

where $u$ and $\bar{u}$ are the complex scalar functions, satisfying the Helmholtz's equation

$$
\frac{\partial^{2} u}{\partial x^{2}}+\frac{\partial^{2} u}{\partial y^{2}}+k^{2} \varepsilon u=0
$$

$k=\omega / c$ is the wave number, $i=\sqrt{ }-1$ is the imaginary unite, $\varepsilon$ is the dielectric permittivity of a medium, which can be a complex value. Here, and everywhere below, in equations hold true for two polarizations $H$ and $E$ simultaneously, the vinculum above the symbol of field function $u$ for the latter is omitted. It is supposed that temporal dependence of fields is determined by the exponential factor $\exp (-i \omega t)$, which is also omitted.

Let the plane wave

$$
u=\exp \left[i k\left(\alpha_{0} x+\beta_{0} y\right)\right]
$$

is incident from negative $x$ and $y$ on the infinitely thin perfectly conducting half-plane $x=0, y \leq 0$ (Fig. 1), where $\alpha_{0}{ }^{2}+\beta_{0}{ }^{2}=\varepsilon$. It is assumed that for the $H$ and $E$ polarizations, the expression (3) determines the $E_{z}$ and $E_{y}$ component of an incident wave, respectively.

We construct the solution of diffraction problem for two semi-infinite domains $x \leq 0$ and $x \geq 0$ separately on the bases of boundary conditions. The tangential components of electric field should be vanish on a perfectly conducting surface, but

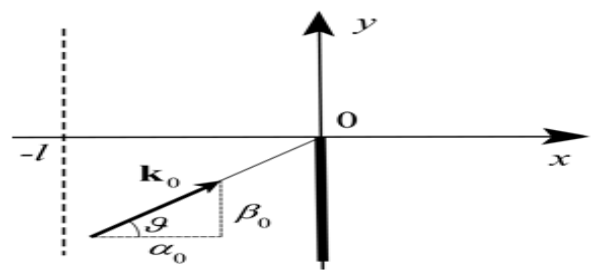

Figure 1. Diffraction of a plane wave by a half-plane.

at its absence, the tangential electric and magnetic components should satisfy the continuity conditions on the boundaries between the indicated domains. From here, using Equations (1), one yields the following boundary conditions for the field functions of the $H$ and $E$ polarizations

$$
\begin{aligned}
& (u)_{x=0}=0 \quad \text { for } y<0 \\
& (u)_{x=-0}=(u)_{x=+0} ;\left(\frac{\partial u}{\partial x}\right)_{x=-0}=\left(\frac{\partial u}{\partial x}\right)_{x=+0} \text { for } \mathrm{y}>0
\end{aligned}
$$

and

$$
\begin{gathered}
\left(\frac{\partial \bar{u}}{\partial x}\right)_{x=0}=0 \quad \text { for } y<0 \\
\left(\frac{\partial \bar{u}}{\partial x}\right)_{x=-0}=\left(\frac{\partial \bar{u}}{\partial x}\right)_{x=+0} ;(\bar{u})_{x=-0}=(\bar{u})_{x=+0} \text { for } \mathrm{y}>0
\end{gathered}
$$

where the symbol " 0 " denotes an infinitesimally small positive value.

To obtain a rigorous solution of diffraction problem, satisfying the Helmholtz's equation (3), one proceeds usually from a superposition of plane waves, what corresponds to decomposition of this solution in a Fourier integral on the initial plane $x=0$. We seek such a solution in the following form, writing that for two polarizations simultaneously

$$
\begin{array}{r}
u=\left\{\exp \left[i k\left(\alpha_{0} x+\beta_{0} y\right)\right] \mp \exp \left[i k\left(-\alpha_{0} x+\beta_{0} y\right)\right]\right\} \alpha_{0}^{-m} \\
\pm \int_{-\infty}^{+\infty} A(\beta) e^{i k(-\alpha x+\beta y)} \alpha^{-m} d \beta \quad \text { for } x \leq 0 \\
u=\int_{-\infty}^{+\infty} A(\beta) e^{i k(\alpha x+\beta y)} \alpha^{-m} d \beta \text { for } x \geq 0
\end{array}
$$

where the upper and lower signs are related to the cases of the $H$ polarization $(m=0)$ and the $E$ polarization $(m=1)$, respectively, $A(\beta)$ is the unknown Fourier image of the diffraction field,

$$
\alpha=\sqrt{\varepsilon-\beta^{2}}
$$

is the value having a nonnegative imaginary part, what should provide nonincreasing field magnitude at moving away from an obstacle (the edge of a half-plane).

Our choice of the field in the form of (6) provides satisfaction of the first conditions (4b) and (5b). Remaining two conditions (4b) and (5b) together with the conditions (4a) and (5a) yield the system of the dual integral equations [2-5]

$$
\int_{-\infty}^{+\infty} A(\beta) e^{i k \beta y} d \beta=0 \quad \text { for } y<0
$$




$$
\int_{-\infty}^{+\infty} A(\beta) e^{i k \beta y} \alpha^{ \pm 1} d \beta-\alpha_{0}^{ \pm 1} e^{i k \beta_{0} y}=0 \quad \text { for } y>0
$$

Let us transform these equations from the spatial domain of the argument $y$ into the spatially-frequency one. For that, we multiply both sides of those by $\exp (-i k \bar{\beta})$ with some $\beta=\bar{\beta}$ and than take integral of the first and second equations along the negative or positive semi axis of the $y$ coordinate, respectively

$$
\begin{gathered}
\int_{-\infty}^{+\infty} A(\beta) \delta_{-}(\beta-\bar{\beta}) d \beta=0 \\
\int_{-\infty}^{+\infty} \alpha^{ \pm 1}\left[A(\beta)-\delta\left(\beta-\beta_{0}\right)\right] \delta_{+}(\beta-\bar{\beta}) d \beta=0
\end{gathered}
$$

where $\delta_{ \pm}(\beta)$ are the generalized functions [18]

$$
\begin{aligned}
& \delta_{+}(z)=\frac{1}{2 \pi} \int_{0}^{+\infty} e^{i z t} d t=-\frac{1}{2 \pi(z+i 0)} \\
& \delta_{-}(z)=\frac{1}{2 \pi} \int_{-\infty}^{0} e^{i z t} d t=\frac{1}{2 \pi(z-i 0)}
\end{aligned}
$$

$\delta(\beta)$ is the routine Dirac delta function [2], for which $\delta_{+}(\beta)+\delta_{-}(\beta)=\delta(\beta)$, and the symbol "0" denotes, as before, an infinitesimally small positive value. If for the certain function $f(\beta)$ the integrals

$$
\begin{aligned}
& f^{-}(\beta)=\int_{-\infty}^{+\infty} f(\bar{\beta}) \delta_{+}(\bar{\beta}-\beta) d \beta \\
& f^{+}(\beta)=\int_{-\infty}^{+\infty} f(\bar{\beta}) \delta_{-}(\bar{\beta}-\beta) d \beta
\end{aligned}
$$

exist, then they determine functions $f^{-}$and $f^{+}$, which are analytic in the lower and upper half-plane of the complex variable $\beta$, respectively, and in the upper (lower) half-plane they have a simple pole. Further, it is obvious, that

$$
\int_{-\infty}^{+\infty} f^{-}(\beta) \delta_{+}(\beta-\bar{\beta}) d \beta=f^{-}(\bar{\beta})
$$

Usually, the transformations (10) are written with the small displacement of the integration pass upper or lower from the real axis, where the poles of integrand are located [2-5]. Utilization of the functions (9) corresponds to the opposite case, when the integration contour are still, but only the poles are subjected to small displacement from the real axis.

Returning to Equations (8), note that from (8a) we yield at once: $A(\beta)=A^{-}(\beta)$, i.e. the function $A(\beta)$ is analytic in the lower complex half-plane and has not poles there. Only for this case the integral in the left side of $(8 a)$ vanishes $[19,20]$. Really, for this integral, the initial contour of integration on the real axis, being completed by a semicircle of infinitely great radius in the lower complex half-plane, turns into a closed pass with analytic integrand in all interior points. Similarly, from (8b) we obtain

$$
\alpha^{ \pm 1}\left(A^{-}(\beta)-\delta\left(\beta-\beta_{0}\right)\right)=B^{+}(\beta)
$$

where $B^{+}(\beta)$ is a certain function, analytic in the upper half-plane. As it is known [2-5], the value $\alpha$ (7) can be represented as a product of two functions $\alpha_{ \pm}$

$$
\alpha=\alpha_{+} \alpha_{-} \quad \alpha_{ \pm}=\sqrt{n \pm \beta}
$$

each of which is analytic in the upper (lower) half-plane of the argument $\beta$, and in the lower (upper) half-plane these functions determine the cuts, passing from the branch point $\beta=\mp n \mp i 0$ to zero and further to the mines (plus) imaginary infinity [4]. Here, $n=\mathcal{E}^{1 / 2}$ is the refractive index of a medium (generally speaking, a complex value). Let us divide (multiply) both sides of (12) on $\alpha_{+}$and apply the first transformation (10) to both sides of the obtained relation:

$$
\int_{-\infty}^{+\infty} \alpha_{-}^{ \pm 1}\left[A^{-}(\beta)-\delta\left(\beta-\beta_{0}\right)\right] \delta_{+}(\beta-\bar{\beta}) d \beta=\int_{-\infty}^{+\infty} \alpha_{+}^{\mp 1} B^{+}(\beta) d \beta
$$

Integrand in the right-hand side is analytic in the upper half-plane, hence, closing the path of integration by a semicircle through this half-plane, we obtain zero result [19, 20]. From here, in view of the property (11), we have

$$
\alpha_{-}^{ \pm 1}(\bar{\beta}) A^{-}(\bar{\beta})-\int_{-\infty}^{+\infty} \alpha_{-}^{ \pm 1} \delta\left(\beta-\beta_{0}\right) \delta_{+}(\beta-\bar{\beta}) d \beta=0
$$

or

$$
A(\beta)=\left(\frac{n-\beta_{0}}{n-\beta}\right)^{ \pm 1 / 2} \delta_{-}\left(\beta-\beta_{0}\right)
$$

This is a solution of diffraction problem in the spatially-frequency domain for two polarizations of field. To obtain the corresponding solution as a function of the spatial coordinates, one should inset (13) into (6) and calculate the diffraction integral

$$
I^{\mp}(x, y)=\left(n-\beta_{0}\right)^{ \pm / 2} \int_{-\infty}^{+\infty} \frac{\delta_{-}\left(\beta-\beta_{0}\right)}{\sqrt{n \mp \beta}} \exp [i k(\alpha|x|+\beta y)] d \beta(14)
$$

For that, consider an auxiliary integral

$$
J^{\mp}(a, b, x, y)=\int_{a}^{b} \exp [i k(\alpha x+\beta y)] \frac{d \beta}{\sqrt{n \mp \beta}}
$$

where $x \geq 0, \alpha=\left(n^{2}-\beta^{2}\right)^{1 / 2}, \operatorname{Im} \alpha \geq 0$. Let us introduce two new variables of integration 


$$
\begin{aligned}
& w(\beta)=\sqrt{\frac{k}{2}}(\sqrt{(n+\beta)(\rho+y)}+\sqrt{(n-\beta)(\rho-y)}) \\
& v(\beta)=\sqrt{\frac{k}{2}}(\sqrt{(n-\beta)(\rho+y)}-\sqrt{(n+\beta)(\rho-y)})
\end{aligned}
$$

where

$$
\rho=\sqrt{x^{2}+y^{2}}
$$

They satisfy the relations

$$
\begin{gathered}
w^{2}-k n \rho=k n \rho-v^{2}=k(\alpha x+\beta y) \\
\frac{d \beta}{\sqrt{n \mp \beta}}=\mp \sqrt{\frac{2}{k}} \frac{1}{\rho}(\sqrt{\rho \mp y} d w \pm \sqrt{\rho \pm y} d v)
\end{gathered}
$$

With their help, the integral (15) is broken up into two different integrals, which are reduced to the complex Fresnel integrals

$$
\begin{gathered}
J^{\mp}(a, b, x, y)=\mp \sqrt{\frac{2}{k}} \frac{1}{\rho}\left\{\sqrt{\rho \mp y} e^{-i k n \rho}\left[\Phi_{+}(w(b))-\Phi_{+}(w(a))\right]\right. \\
\left. \pm \sqrt{\rho \pm y} e^{i k n \rho}\left[\Phi_{-}(v(b))-\Phi_{-}(v(a))\right]\right\}
\end{gathered}
$$

where

$$
\Phi_{ \pm}(z)=\int_{0}^{z} \exp \left( \pm i t^{2}\right) d t
$$

Let us evaluate the integral (17) at $a \rightarrow-\infty, b \rightarrow+\infty$. Since at that $\pi / 4 \leq \arg (w(a)) \leq \pi / 2,0 \leq \arg (w(b)) \leq \pi / 4$, and $-\pi / 4 \leq \arg (v(a)) \leq 0, \pi / 2 \leq \arg (v(b)) \leq 3 \pi / 4$, then the first expression in the square brackets vanishes, and the second one equals to $\pm(\pi / 2)^{1 / 2}(1-i)$, so that

$$
J^{\mp}(-\infty,+\infty, x, y)=\frac{1-i}{\rho} \sqrt{\frac{\pi(\rho \pm y)}{k}} e^{i k n \rho}
$$

Now, let us rewrite the initial diffraction integral (14) in the form

$$
I^{\mp}(x, y)=\frac{\left(n-\beta_{0}\right)^{ \pm 1 / 2}}{2 \pi i} \exp \left[i k\left(\beta_{0}+i 0\right) y\right] S^{\mp}(x, y)
$$

where

$$
S^{\mp}(x, y)=\int_{-\infty}^{+\infty} \frac{\exp \left\{\left[i k\left[\alpha|x|+\left(\beta-\beta_{0}-i 0\right) y\right]\right\}\right.}{\sqrt{n \mp \beta}\left(\beta-\beta_{0}-i 0\right)} d \beta
$$

For computation of this integral, let us apply the method of differentiation with respect to parameter [20]:

$$
\frac{\partial}{\partial y} S^{\mp}(x, y)=i k e^{-i k\left(\beta_{0}+i 0\right) y} J^{\mp}(-\infty,+\infty,|x|, y)
$$

$$
=\frac{(1+i)}{\rho} \sqrt{\pi k(\rho \pm y)} \exp \left\{i k\left[\rho-\left(\beta_{0}+i 0\right) y\right]\right\}
$$

from which

$$
S^{\mp}(x, y)=(1+i) \sqrt{\pi k} \int_{-\infty}^{y} \sqrt{\rho \pm y} \exp \left\{i k\left[\rho-\left(\beta_{0}+i 0\right) y\right]\right\} \frac{d y}{\rho}(20)
$$

The lower limit of integration is taken as $-\infty$, because for this value of $y$ exactly the integrand of (19) vanishes. This conclusion is true as for complex values of the propagation parameter $\beta_{0}$ (with a positive imaginary part), as for real its values owing to the presence of infinitely small additive $i 0$ to the value $\beta_{0}$. To compute the integral (20), one can use the variables $p=w\left(-\beta_{0}\right)$ and $q=-v\left(\beta_{0}\right)(16)$, but now, as functions of the $y$ coordinate. Note that

$$
\begin{gathered}
p^{2}-k \alpha_{0}|x|=q^{2}+k \alpha_{0}|x|=k\left(n \rho-\beta_{0} y\right) \\
\sqrt{\rho \pm y} \frac{d y}{\rho}= \pm \sqrt{\frac{2}{k}} \frac{d p \mp d q}{\sqrt{n \mp \beta_{0}}}
\end{gathered}
$$

These relations allow to express the integral (20) in terms of the Fresnel integrals. As a result, we obtain for the integral (18)

$$
I^{\mp}(x, y)=\left(e^{i k \alpha_{0}|x|} F(q) \mp e^{-i k \alpha_{0}|x|} F(p)\right) e^{i k \beta_{0} y}
$$

where

$$
F(z)=\frac{1-i}{\sqrt{2 \pi}} \int_{z}^{+\infty} e^{i t^{2}} d t
$$

is the additional complex Fresnel integral. A constant multiplier before that normalizes its value in infinity: $F(-\infty)=1$.

Thereby we have calculated the diffraction integral (14) of the problem actually without extending on a complex plane of the integration argument $\beta$. Such a method of computation are more simple than the usual method [2-5], which requires extension on a complex plane, but gives result, valid only for real parameters of incident wave propagation.

For the case $x \geq 0$ the expression (21) at once gives the field function (6b). But for $x \leq 0$ one should add exponential terms to this expression. Taking into account that $|x|=-x$, and converting from modulus of this coordinate to its real value, we have

$$
u=\alpha_{0}^{-m} D^{\mp}\left(\beta_{0}, x, y\right)
$$

where $m=0$ for the $H$ polarization and $m=1$ for the $E$ polarization,

$$
\begin{gathered}
D^{\mp}\left(\beta_{0}, x, y\right)=F(q) \exp \left[i k\left(\alpha_{0} x+\beta_{0} y\right)\right] \\
\mp F(p) \exp \left[i k\left(-\alpha_{0} x+\beta_{0} y\right)\right]
\end{gathered}
$$




$$
\left\{\begin{array}{l}
p \\
q
\end{array}\right\}=\sqrt{\frac{k}{2}}\left(x \sqrt{\frac{n+\beta_{0}}{\rho+y}} \pm \sqrt{\left(n-\beta_{0}\right)(\rho+y)}\right)
$$

To obtain all components of the total field, one should insert the expressions (22)-(24) into (1) and calculate spatial derivatives. As a result, we give

\section{$H$ polarization}

$$
\begin{aligned}
& E_{z}=D^{-}\left(\beta_{0}, x, y\right) \\
& H_{x}=\beta_{0} D^{-}\left(\beta_{0}, x, y\right)-\frac{1+i}{2 \rho} \sqrt{\frac{\left(n-\beta_{0}\right)(\rho+y)}{k \pi}} e^{i k n \rho} \\
& H_{y}=-\alpha_{0} D^{+}\left(\beta_{0}, x, y\right)-\frac{1+i}{2 \rho} x \sqrt{\frac{n-\beta_{0}}{k \pi(\rho+y)}} e^{i k n \rho}
\end{aligned}
$$

\section{E polarization}

$$
E_{x}=-\frac{\beta_{0}}{\alpha_{0}} D^{+}\left(\beta_{0}, x, y\right)+\frac{1+i}{2 \rho} \frac{x}{\sqrt{k \pi\left(n-\beta_{0}\right)(\rho+y)}} e^{i k n \rho}
$$

$$
E_{y}=D^{-}\left(\beta_{0}, x, y\right)+\frac{1+i}{2 \rho} \sqrt{\frac{\rho+y}{k \pi\left(n-\beta_{0}\right)}} e^{i k n \rho}
$$

$$
H_{z}=D^{+}\left(\beta_{0}, x, y\right) \mathcal{\varepsilon} / \alpha_{0}
$$

If $n=1$ and $\beta_{0}{ }^{2}<1$, then the parameters of propagation of an incident wave $\alpha_{0}$ and $\beta_{0}$ can be characterized by sine and cosine of the angle $\psi$, determining the direction of their incidence relative to the plane $x=0: \alpha_{0}=\sin \psi, \beta_{0}=\cos \psi$ (in [2], this angle is denoted as $\alpha_{0}$ ). The coordinates $x$ and $y$ of an observation point can be also determined in terms of the value $\rho=\left(x^{2}+y^{2}\right)^{1 / 2}$ and orientation angle of the radius-vector of the given point $\varphi: x=-\rho \sin \varphi, y=-\rho \cos \varphi$ (for this angle, corresponding designation in [2] is $\theta$ ). Then

$$
\begin{gathered}
\pm \alpha_{0} x+\beta_{0} y=-\rho \cos (\psi \mp \varphi) \\
p=-\sqrt{2 k \rho} \cos (\psi+\varphi) \quad q=-\sqrt{2 k \rho} \cos (\psi-\varphi)
\end{gathered}
$$

and the solution of diffraction problem (25), (26), written in terms of two angle parameters $\psi$ and $\varphi$, coincides with the solution, presented in [2], up to the constant factor $\alpha_{0}^{-1}$ for the $E$ polarization.

The terms proportional to $\exp (i k \rho)$ in the right-hand sizes of (25b), (25c) and (26a), (26b), determine singular edge waves, whose amplitudes behave as $\rho^{-1 / 2}$ at $\rho \rightarrow 0$, i.e. increase infinitely at nearing to the edge of a half-plane. However, as it is well-known, such an increase of amplitudes does not contradict to the physical requirement of finiteness of electromagnetic energy magnitude in any finite spatial domain without sources, because corresponding volume integrals for fractional negative dependence on the distance take on finite values.

\section{Calculation Results}

If the passing from the general solution (25), (26) to the particular Sommerfeld's solution, presenting in [2-5], is sufficiently straightforward, the inverse passing is not presented as fully obvious. The field of application of the solution (25), (26) is more wide than that for the standard solution [2-5], written in terms of angular variables and justified for real values of those. Firstly, the solution (25), (26) admits complexity of the refractive index of a medium $n$ Secondly, this solution, being expressed explicitly in terms of parameters of incident wave propagation $\alpha_{0}$ and $\beta_{0}$, admits their complexity, i.e. allows to study diffraction of decaying and inhomogeneous waves. To demonstrate this feature of our solution by means of numerical computations, we consider
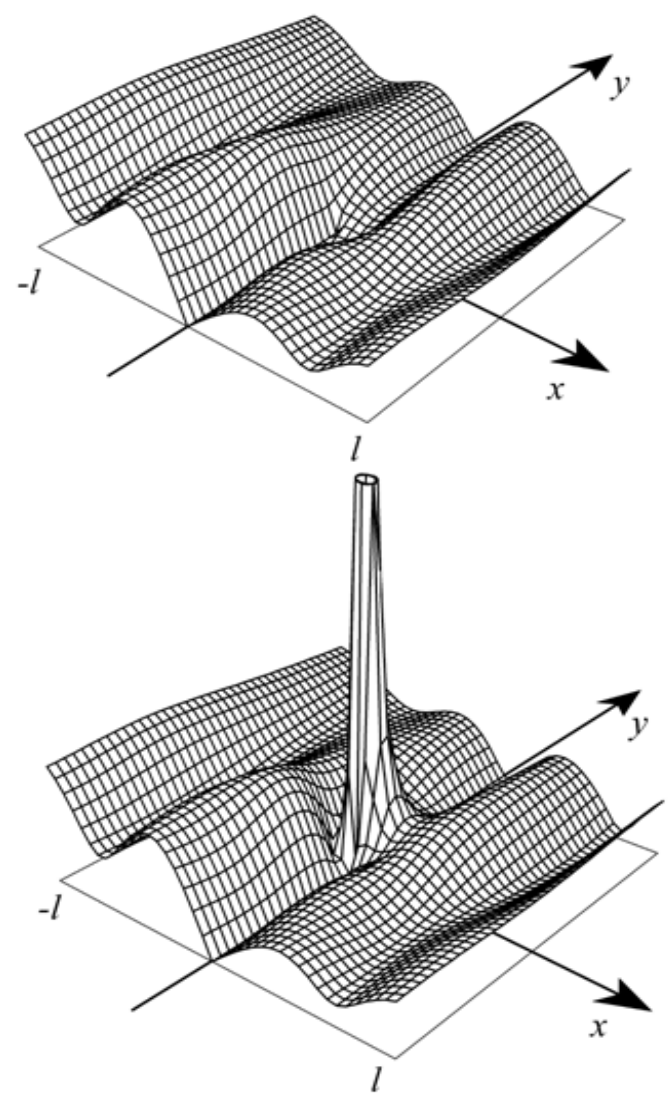

Figure 2. Spatial distribution of electric field magnitude for the components $E_{z}$ (the upper part) and $E_{y}$ (the lower part), when inhomogeneous plane waves of various polarizations diffract by a perfectly conducting half-plane in an absorbing medium.

the cases of real $\beta_{0}\left(\beta_{0}=\sin \vartheta, \vartheta\right.$ is the angle of incidence, Fig. $1)$. This corresponds to excitation of the given wave after refraction on a certain plane boundary $x=-l$ with a certain transparent medium, which is located at $x<-l$. It is obviously, that here only one parameter of propagation $\alpha_{0}=\left(\varepsilon-\beta_{0}{ }^{2}\right)^{1 / 2}$ can be complex-valued, having a nonnegative imaginary part, what should provide nonincreasing wave amplitude at moving away from a source. 

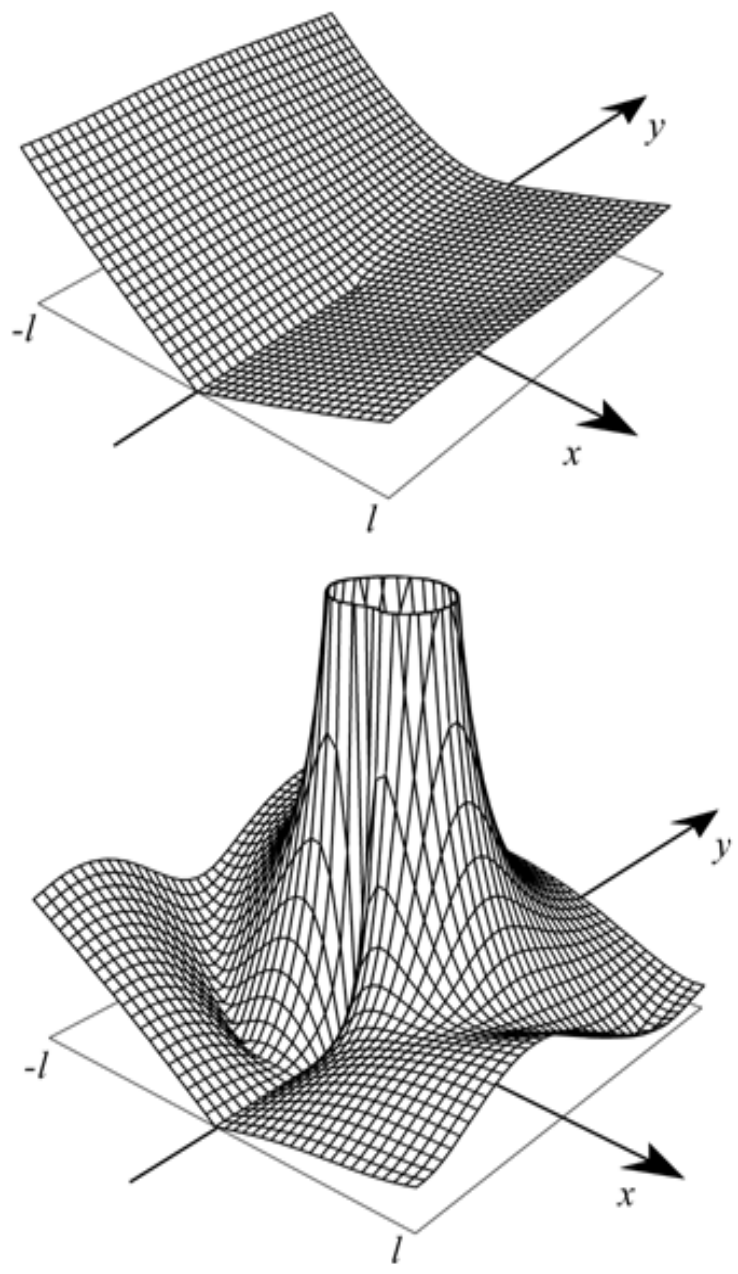

Figure 3. Spatial distribution of electric field magnitude for the components $E_{z}$ (the upper part) and $E_{y}$ (the lower part), when decaying plane waves of various polarizations diffract by a perfectly conducting half-plane in a transparent medium.

Figs. 2 and 3 displays results of computations according Equations (25) and (26) for magnitudes of two electric field components $E_{z}$ and $E_{y}$ under the diffraction of an imhomogeneous plane wave of the $H$ or $E$ polarization by a conducting half-plane, surrounded by a transparent and absorbing medium at $l=3.2 / \mathrm{k}$. In the first case (Fig. 2), $n=1.5+0.21 i, \beta_{0}=0.5$, and the incident wave decays in the $x$ direction. In the second case (Fig. 3 ), $n=1$, but $\beta_{0}=1.024$, so that this wave also decays in the same direction with a such coefficient of decay in magnitude. The difference between these two cases displays in the presence of phase variations along this direction in an absorbing medium. For

the case of the $E$ polarization, the influence of a singular edge wave is added to that. The amplitude of this wave is depended essentially on difference between the value $\beta_{0}$ and the refractive index $n$. For the case of excitation by total internal reflection (Fig. 3), rather wide domain of increase for the field amplitude near the half-plane edge is caused by small difference between $\beta_{0}=1.024$ and $n=1$. For comparison, we present on Fig. 4 analogous computation results for the classical case of the homogeneous wave
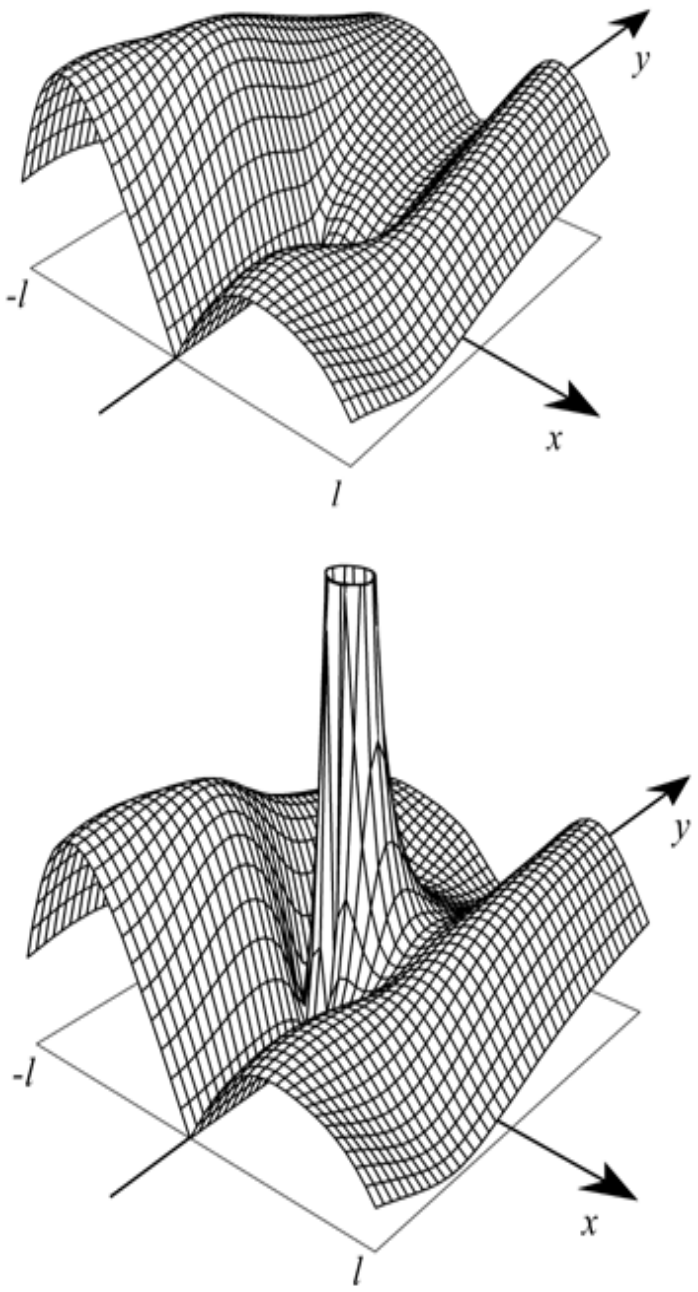

Figure 4. Spatial distribution of electric field magnitude for the components $E_{z}$ (the upper part) and $E_{y}$ (the lower part), when homogeneous plane waves of various polarizations diffract by a perfectly conducting half-plane in a transparent medium.

diffraction [2-5] at $n=1, \beta_{0}=0.5$.

Similarly, one can study the other case of excitation of the initial wave (3) after refraction on the dielectric interface $y=l$, parallel to the $x$ axes. Then $\alpha_{0}=\cos \vartheta$, and $\beta_{0}=\left(\varepsilon-\alpha_{0}^{2}\right)^{1 / 2}$ should have a nonpositive imaginary part.

\section{Conclusion}

The solution of the Sommerfeld's diffraction problem for a homogeneous incident wave can be generalized on the cases of an inhomogeneous decaying wave and of an absorbing medium, surrounding the half-plane, if one considers the problem in terms of parameters of propagation of an incident wave instead of its angle of incidence. The formal rewriting of the standard Sommerfeld's solution on complex domain of propagation parameters and of refractive index of medium, is not too difficult, but at that, one should prove validity of such a solution, since the initial Sommerfeld's solution was grounded only for their real values. In this work, we prove validity of our general solution by a nonstandard technique, which does not use any 
assumptions about reality of propagation para meters and of the refractive index of a medium. Besides, this technique utilizes generalized functions and does not use a complex argument for diffraction integrals computation. Perhaps, the given technique with some modifications will be useful for obtaining rigorous solutions of another similar diffraction problems.

\section{References}

[1] A. Sommerfeld, "Mathematische Theorie der Diffraktion," Mathematische Annalen, vol. 47, pp. 317-374, 1896 (in German).

[2] M. Born, and E. Wolf, Principles of Optics, 7th ed., Cambridge, Cambridge University Press, 1999.

[3] L. A. Weinstein, The Theory of Diffraction and the Factorization Method, Boulder, Golem, 1969.

[4] R. Mittra, and S. W. Lee, Analytical Techniques in the Theory of Guided Waves, New York, Macmillan, 1971.

[5] B. Noble, Methods Based on the Wiener-Hopf Technique for the Solution of Partial Differential Equations, London, Pergamon, 1958.

[6] Y. Z. Umul, "Scattering of a Gaussian beam by an impedance half-plane," JOSA A, vol. 24, issue 10, pp. 3159-3167, 2007.

[7] S. P. Anokhov, "Plane wave diffraction by a perfectly transparent half-plane," JOSA A, vol. 24, issue 9, pp. 2493-2498, 2007.

[8] A. Ciarkowski, "Electromagnetic pulse diffraction by a moving half-plane," Progress In Electromagnetics Research, vol. PIER 64, pp. 53-67, 2006.

[9] W. Hussain, "Asymptotic analysis of a line source diffraction by a perfectly conducting half-plane in a bi-isotropic medium," Progress In Electromagnetics Research, vol. PIER 58, pp. 271-283, 2006.

[10] Y. Z. Umul, and U. Yalçın, "Scattered fields of conducting half-plane between two dielectric media," Applied Optics, vol. 49, issue 20, pp. 4010-4017, 2010.

[11] K. L. McDonald, "Diffraction of spherical vector waves by an infinite half-plane," JOSA, vol. 43, issue 8, pp. 641-646, 1953.

[12] Y. Luo, and B. Lü, "Polarization singularities of Gaussian vortex beams diffracted at a half-plane screen beyond the paraxial approximation," JOSA A, vol. 26, issue 9, pp. 1961-1966, 2009.

[13] Y. Z. Umul, "Uniform theory for the diffraction of evanescent plane waves," JOSA A, vol. 24, issue 8, pp. 2426-2430, 2007.

[14] Y. Z. Umul, "Diffraction of evanescent plane waves by a resistive half-plane," JOSA A, vol. 24, issue 10, pp. 3226-3232, 2007.

[15] Y. Z. Umul, "Diffraction by a black half plane: Modified theory of physical optics approach," Optics Express, vol. 13, No. 19, pp. 7276-7287, 2005.

[16] J. B. Schneider, and R. J. Kruhlak, "Dispersion of homogeneous and inhomogeneous waves in the Yee finite-difference time-domain grid," IEEE Trans. Microwave Theory \& Techn., vol. 49, No. 2, pp. 280-287, 2001.

[17] J. A. Stratton, Electromagnetic Theory, New York, McGraw-Hill, 1941.

[18] N. N. Bogolyubov, and D. V. Shirkov, Introduction to the Theory of Quantized Fields, New York, Wiley, 1980.

[19] E. C. Titchmarsh, Theory of Functions, Oxford, Oxford University Press, 1976.

[20] A. G. Sveshnicov, and A. N. Tikhonov, The Theory of Functions of a Complex Variable, Moscow, Mir Publ., 1978. 